\title{
Über die Existenz eines gasförmigen Hydrides von Thorium.
}

\author{
Von Albert Krauber und Julius Mell von Mellenhetm.
}

\section{(1. Mitteilung.)}

Die ersten Untersuchungen über ein festes Hydrid von Thorium verdanken wir CLEMENS WINKLER ${ }^{1}$ ), welcher den Nachweis erbrachte, dab sich bei Einwirkung von Wasserstoff in statu nascendi auf metallisehes Thorium ein Produkt bildet, dessen Konstitutionsformel $\mathrm{ThH}_{2}$ sich annähernd aus der Analyse ergab. Er erhitzte zwecks Darstellung dieser Verbindung ein Gemenge bestehend aus 1 Mol. $\mathrm{ThO}_{2}$ mit 2 At. Magnesium plus $20 \%$ Überschuß in einem einseitig verschlossenen Rohr im Wasserstoffstrom, unter mäßiger Erhitzung und erhielt ein Reaktionsprodukt von grauschwarzer Farbe. Wurde Magnesium im Überschuß benutzt, so resultierte nach seinen Angaben eine Masse mit etwa 0,50\% H-Gehalt, während bei Anwendung gleicher Teile der beiden Stoffe ein braungrauer Körper mit etwa 0,13\% H-Gehalt entstand. Das Hydrid, als welches das grauschwarze Reaktionsprodukt angesprochen wurde, ist bei höherer Temperatur leicht entzündlich and verbrennt im Sauerstoffstrom unter blendender Lichterscheinung. Bei der Analyse konstatierte er, daß der Vorgang nicht vollständig nach der Formel

$$
\mathrm{ThO}_{2}+2 \mathrm{Mg}+2 \mathrm{H}=\mathrm{ThH}_{2}+2 \mathrm{MgO}
$$

seinen Verlauf nimmt.

Die Aufnahmefähigkeit des metallischen Thoriums für Wasserstoff wurde von C. MAtignoN ${ }^{2}$ ) bestätigt. Festes Thorhydrid soll

1) CL. WrNkLeR, Ber. 1891, 873-899; J. Berz. 1891, 494.

$\left.{ }^{2}\right)$ C. Matranon, Compt. rend. 131 (1900), 891; Chem. Centralbl. (1901), I, 85; Chem. Zig. 24, 1062. 
nach Wiakler, Matignon and Delepine ${ }^{1}$ ), mit Salzsäure übergossen, Wasserstoff liefern.

Letztgenannte fanden jedoch für dessen Zusammensetzung die Formel $\mathrm{ThH}_{4}$. E. Chauvenet ${ }^{2}$ ) stellte festes Thorhydrid nach er Güntschen Methode aus

$$
4 \mathrm{LiH}+\mathrm{ThCl}_{4}=4 \mathrm{LiCl}+\mathrm{ThH}_{4}
$$

dar. Auch für diese Formel konnte keine vollkommene Übereinstimmung mit den berechneten Resultaten erlangt werden. ${ }^{3}$ )

Nach der Entdeckung der letzten unbekannten gasförmigen Wasserstoffverbindungen der Gruppe IV des periodischen Systems durch Fritz Paneth und seiner Mitarbeiter, dank der besonders verfeinerten Apparatur and Einführung neuer Arbeitsmethoden in die "Wasserst offchemie " durch genannten Forscher ${ }^{4}$ ), hielt man auch in der Gruppe IVa Umschau und die Mitteilung von R. Schwarz und H. DeIsLeR ${ }^{5}$ spricht bereits von einem gasförmigen Zirkonwasserstoff. Sie wiesen nach, daB bei der Reduktion von $\mathrm{ZrO}_{\mathbf{a}}$ mit Magnesium ein eigentlicher Körper von der Zusammensetzung des Zirkonmonoxydes nicht entsteht, hingegen elementares Zirkon, mit unverändertem Zirkondioxyd, sowie geringe Mengen eines Magnesiumzirkonides gebildet werden. Mit konzentrierter Salzsäure übergossen, lieferte die Zirkonmasse ein oft zur Selbstentzündung neigendes Gas, welches an einem zu einer Spitze ausgezogenen Glasrohr einen Beschlag von Zirkondioxyd absetzte. Dieses Gas wurde als Zirkonwasserstoff identifiziert.

Oblgeich die Zahl der bislang genau charakterisierten Legierungen von Thorium gering ist, es sind dies die Thor-Silizium ${ }^{6}$ ) (ThSi) und

1) C. Matignon und M. Drafépine, Compt. rend. 132, 37; Chem. Centralbl. (1901), J, 295; A.ch. [8], 10, 139; Chem. Centralbl. (1907) I, 1022.

2) E. Chadvinht, Chem.-Ztg. 34, 940.

s) Die e ngehende Besprechung dit ser Arbeiten wollen wir erst in der zweiten Mitteilung vol nehmen.

4) F. Paneth u. K. Fürth, Ber. 52 (1919), 2020 u. 2029; Paneth und E. Winterniqu, Ber. 51 (1918), 1704 u. 1728; PANETH, Wiener Ber. II a, 127 (1918), $1729-1761$.

5) R. Schwarz u. H. Deisler, Ber. 52 (1919), 1900.

6) Moissan III, 88; E. Whdekind und K. Fetzer, Chem.-Ztg. 29, 1032; Chem. Centralbl. (1905) II, 1224; Konsort. für elektrochem. Ind. (D. R. P. 164990 and $166374 \mathrm{Kl} .21 \mathrm{~h}$; Chem. Centralbl. (1900) II, 1760; eb ndort (1906) I, 518 . 
Thorplatinlegierung ${ }^{1}$ ) (ThPt), ferner $\left.\left.\mathrm{Ih}_{y} \mathrm{P}_{y}{ }^{2}\right), \mathrm{Th}_{x} \mathrm{Se}_{y}{ }^{3}\right) \mathrm{Th}_{x} \mathrm{~N}_{y}{ }^{3,4}, 8$ ), $\left.\mathrm{Th}_{x} \mathrm{C}_{y}{ }^{5}\right), \mathrm{Th}_{x} \mathrm{Hg}_{y}{ }^{6}$ ), lag es doch auf der Hand, auch die Existenz einer Thor-Magnesiumlegierung $\mathrm{ThMg}_{2}$ anzunehmen, wenn diese auch nur in geringen Mengen gebildet wird. Für ein gasförmiges Hydrid von Thorium $\left(\mathrm{ThH}_{4}\right)$ sprechen neben den wenig erforschten Legierungen mit Metallen in erster Linie seine große Affinität zu Wasserstoff als auch die Verbindungen mit den Halogenen, welche sämtlich den Formeltypus $\operatorname{ThX}_{4}$ (X-Halogen) ${ }^{7-14}$ ) aufweisen, sowie die zahlreichen Additionsprodukte mit Ammoniak und organischen Basen.4)

1) L. Nizhson, Ber. 1883, 153; J. Berz. 1883, 118 u. 409.

ग) Berzelus, K. Vet. Acad. Handl. 1829, 1; Ann. d. Phys. 16, 385; J. Berz. 1881, 98. l, 834.

3) Morasan und Etard, Compt. rend. 122, 573-577; Chem. Centrabbl. (1896)

4) Morssan und Martinsfan, Compt. rend. 140, 1512; Chem. Centrabbl. (1905), II, 208. Chauvener, Compt. rend. 151, 389 ; Chem. Centralbl. (1910) II, 1027 .

8) W. L. VomLker, D.R.P. 109864 und 111481, Kl. 11 [1898]; L. Wnass, D.R.P. 129416, Kl. 12m; Chem. Centralbl.(1902) I, 899 und GasBer, 46 (1902), 809; Srmmens u. Harske. D.R.P. 140503, 153352 und 158571, Kl. h 1 (1902/3);

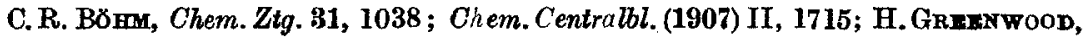
Soc. 93, 1493; Chem. Centralbl. (1908) II, 1156.

6) W. KеттвмBвIx, Z. anorg. Chem. 38 (1904), 217; Chem. Centralbl. (1904) I, 503. Dieser konnte die Verbindung nioht erhalten.

7) Troost u. Otvrard, Ann. chim. phys. (6) 17, 227-246; Chem. Centralbl. 1889, II, 20.

s) J. J. Chydeñus, Kemisk undersökning of Thorjord ock Thorsalter, Helsingtors 1861; Ann. d. Phys. 119, 43; J. Berz. 1863, 194.

9) J. Mrenit u. Matthews, Journ. Amer. Chem. Soc. 20, 819-843; Chem. Centralbl. 1891, 15-16.

10) KRÜss u. NrLson, Ofvers. af $K$. Swenska Vetenskaps Acad. Förhandlinger 1887, 977; Z. phys. Chem. 1, 301; J. Berz. 1887, 70.

11) NrusoN, Ber. 1882, 2537; C. R. 95, 727; J. Berz. 1882, 352.

12) Edgar F. Smith u. Harrife, Journ. Am. Chem. Soc. 17, 654-656; Chem. Centralbl. 2 (1895), 590.

13) P. J 283-287; Chem. Centralbl. 1894, I, 13; Lasinsky u. Gundutor, ebenda 15, 81-83; Chem. Centralbl. 1897, II, 790.

16) O. Hösresohmid und ST. Hoвotitx, Wien. Ber. 125, II* (1916), 149 . 
Der Umstand, daß das Thorium vermöge seiner radioaktiven Eigenschaften eine Ausnahmestellung verdient, sich somit, mit den für die Bildung der Wasserstoffverbindungen notwendigen Elektronen beladen könne, um daher als negativer Bestandteil zu reagieren, sprach für die Existenz des Thoriumwasserstoffes. Allerdings muBten wir seine Unbeständigkeit vorher ins Auge fassen, zumal seine Bildung aus der Lösung des Chlorides durch naszierenden Wasserstoff ausgeschlossen war. Unter Berücksichtigung der gröBten VorsichtsmaBregeln gelang es dennoch, den Thorwasserstoff zu erhalten und nach den in der Folge wiedergegebenen Methoden sicherzustellen.

\section{Identifizierung des Thoriumwasserstoffes und die Arbeitsmethoden der Wasserstoffohemie.}

Die auf diesem Gebiete allgemein üblichen Methoden machten auch wir uns zunutze, indem wir durch Reduktion von Thoriumdioxyd mit Magnesium zunächst metallisches Thorium, durch weitere Einwirkung von Magnesium die Verbindung $\mathrm{ThMg}_{2}$ anstrebten. Dies bezweckend, erhitzten wir Thorium oxydatum anhydr. $\left(\mathrm{ThO}_{2}\right)$ (MERoksches Präparat) mit möglichst Silicium-freiem wenig oxydiertem Magnesium pulvis. Merck im Gewichtsverhältnis 2:4 oder 2:5 in einem Roseschen Eisentiegel, während der Zeitdauer von 45 Minuten unter konstantem Einleiten eines vollkommen gereinigten kräftigen Wasserstoffstromes zu dunkler Rotglut. Die resultierende Reaktionsmasse war je nach der Zusammensetzung and Erhitzungsdauer mehr oder weniget schwarzbraun bis grau gefärbt. Die metallisch aussehenden, am Boden des Tiegels vorgefundenen Körper wiesen die Eigenschaften einer Legierung dieser beiden Metalle auf. Vorhersschend war aber jene graubraune Masse mit dieser bekamen wir die günstigste Ausbeute -, welche noch warm in der Reibschale pulverisiert und im Exsikkator aufbewahrt wurde. Dabei machte sich ein charakteristischer Geruch bemerkbar, der nachweislich durch die Feuchtigkeit der Luft entstand und von dem gebildeten Hydrid herrührte. Das schwarzgraue Produkt entwickelte Ammoniak, wahrscheinlich durch Bildung des Magnesiumnitrides. Wurde die braungraue Masse mit Wasser übergossen, so entwickelte sich bereits das Gas, beim Übergießen mit verdünnten Säuren nahm die Reaktion sehr stürmischen Verlauf. Der Thorwasserstoff neigt zur Selbstentzündung und es ist daher bei der Arbeit mit diesem Gase Vorsicht geboten. 
Zur Entwicklung des gasförmigen Thorhydrides bedienten wir uns der von Srock und Doнт angegebenen ${ }^{1}$ ), von PANETH ${ }^{2}$ ) verbesserten Apparatur, welche aus einer dreifach tubulierten etwa $500 \mathrm{ccm}$ fassenden WULFeschen Flasche mit Tropftrichter, Zuleitung für diè Legierung, Ableitungsröhre für das gebildete Gas und einem am Boden des Gefäßes befindlichen Abfluß bestand, welcher dazu diente, um während der Arbeit die Säure im Entwicklungsgefäß ablassen, durch den Tropftrichter frische Säure zuleiten zu können, aber auch bei Überdruck als Sicherheitsventil zu funktionieren. Durch das Entwicklungsgefäß wurde ein vollkommen gereinigter aus chemisch reinem Zink und verdünnter Schwefelsäure gewonnener Wasserstoffstrom derart geleitet, daß die Zuleitung einerseits durch den Tropftrichter erfolgte, der für diese Zwecke ein seitlich angeschmolzenes Glasrohr trug, andererseits von einem zweiten Kıppschen Apparat über das tulpenförmig geformte Zufuhrgefäß, in welchem die Legierung eingebracht wurde, strich. Letztere wurde dadurch vor der sonst unvermeidlichen Zersetzung bewahrt. Zunächst brachten wir hinter der Entwicklungsflasche ein mit Chlorcalcium gefülltes U-Rohr an, an dessen Zuleitung eine Glaskugel angeschmolzen war, um die verspritzte Säure abzufangen und unschädlich zu machen. In der U-Röhre selbst wurde aus gleichem Grunde ein dünnes Wattefilter vorgesehen. Die weitere Verbindung bildete ein mit Phosphorpentoxyd gefülltes Kugelrohr. Zur Erzeugung des Spiegels diente ein $3-5 \mathrm{~mm}$ weites unverjüngtes Hartglasrohr. Die Mündung des $\mathrm{P}_{2} \mathrm{O}_{5}$-Rohres gegen die Marshsche Röhre war gleichfalls durch ein dünnes Wattefilter abgesperrt. Dünne Wattefilter sind deshalb empfehlenswert, da wir wiederholt den zerstörenden Einfluß dichter Wattepfropfen auf das Thorgas beobachten konnten. Letzten Endes wurde an die Zersetzungsröhre noch ein in eine Spitze ausgezogenes, mit Glashahn versehenes Rohr angeschaltet und daselbst der Wasserstioff angezündet.

Von der Reinheit des benutzten Materiales überzeugten wir uns stets durch Blindversuche, indem der Brenner unterhalb der Zersetzungsröhre angezündet und 10-15 Minuten erhitzt wurde. Es bildete sich meistens nur ein schwacher, sofort hinter der Erhitzungsstelle abgelagerter Siliciumspiegel von weißer Farbe, neben einem

1) A. Stock u. W. Doнт, Ber. 35 (1902), 2270.

)) F. Ралеth u. Fükth, 1. c., 2023. 
schwach grauen Anflug von Arsen, welcher aus der im Entwicklungsgefäß befindlichen Salzsäure stammte. Die Konzentration der Salzsëure war 6- $n$. Nachdem wir uns überzeugt hatten, daß bei dieser Konzentration die günstigste Ausbeute zu erzielen war, verblieben wir auch bei den später zu besprechenden Kondensationsversuchen dabei.

Der angesetzte Arsenspiegel wurde durch Erhitzen mit dem Brenner verflüchtigt und sodann mit dem Eintragen der Reaktionsmasse begonnen. Nach Verlauf einiger Minuten (meist 5-10, abhängig von der Stärke des Wasserstoffstromes und dem Grad der Erhitzung, deren Intensität wir auf höchster Stufe erhielten) setzte sich unweit der Erhitzungsstelle ein brauner, scharf ausgeprägter Ring ab, der in Schwarzbraun, Braun überging und allmählich verlief, gegen den erhitzten Teil der Röhre dunkelgraue Ablagerungen von metallischem Aussehen aufwies. Der braune Ring konnte durch krïftiges Erhitzen in einen grau gefärbten übergeführt werden.

Diese Beobachtung kann folgendermaßen gedeutet werden: Die Ablagerung des braunen und grauen Ringes, sowie die Umwandlung des ersteren in die graue Form, spricht für die Existenz zweier Modifikationen des Thoriums von unbekanntem Umwandlungspunkt. Das graue kristallinische Thor weist, unter dem Mikroskop betrachtet, motallischen Glanz und Lamellenstruktur auf, während die braune Modifikation amorph erscheint. Eine Verwechslung mit dem gleichfalls dimorphen Silicium ist ausgeschlossen, da selbiges stets als weißer Ring unmittelbar hinter der Erhitzungsstelle erscheint. Für die Erzeugung von Thorspiegeln ist es stets notwendig, die leichte Zer:etzlichkeit des Thorwasserstoffes im Auge zu behalten und die Zuleitung von dem Fntwicklungsgefäß möglichst kurz zu wählen. Was die Temperatur angeht, bei welcher das Hydrid gebildet wird, so ist diese zwischen $15-20^{\circ} \mathrm{C}$ günstig. An heißen Julitagen waren stets ungünstige Resultate zu verzeichnen. Das gleiche gilt von Eiskühlung. Die Stärke des Gasstromes ist auf höchster Stufe zu erhalten, das Eintragen der Reaktionsmasse soll in regelmäßigen Abständen erfolgen. Wir machen alle diese Angaben deshalb, um die Kontrolle unserer Daten leichter durchzuführen.

Die Farbe der von den Metallen As, Sb, Bi, Sn, Si im Vergleich zu dem von Thorium hervorgebrachten Spiegel sei nun der besseren Übersicht halber tabellarisch zusammengefaßt. 


\begin{tabular}{|c|c|c|c|c|}
\hline \multirow{2}{*}{$\frac{\text { Metall }}{\text { Arsen }}$} & \multicolumn{4}{|c|}{ Farbe des Spiegels } \\
\hline & & braungrau & $\begin{array}{c}\text { schwarzbraun } \\
\text { metalliseh }\end{array}$ & $\begin{array}{l}\text { braungelb, } \\
\text { allmählich verlaufend }\end{array}$ \\
\hline Antimon & & $\begin{array}{l}\text { ochwarzgrau } \\
\text { (als Haupt- } \\
\text { bestandteil) }\end{array}$ & $"$ & $"$ \\
\hline Wismut & weiBlichgrau & $\because$ & $n$ & $"$ \\
\hline Zinn & $"$ & $"$ & $n$ & $\eta$ \\
\hline Silicium & weiB & $"$ & $\because$ & $"$ \\
\hline Thorium & $\begin{array}{c}\text { dunkelgrau } \\
\text { metalliseh }\end{array}$ & schwarzgrau & braun & $\begin{array}{l}\text { braungrau, } \\
\text { allmsiblich verlaufend }\end{array}$ \\
\hline
\end{tabular}

Aus der Tabelle geht hervor, $\mathrm{daB} \mathrm{Sn}, \mathrm{Bi}$ und $\mathrm{Th}$ in bezug auf Farbe des Spiegels sehr ähnlich sind; man wird sich daher zur Unterscheidung dieser Metalle der spezifischen Reaktionen bedienen. Ein Arsenspiegel, der aus der zur Zersetzung angewandten Salzsäure entstanden sein könnte, wird von Thorium leicht unterschieden, da, wie schon erwähnt, Arsenspiegel sich durch Erhitzen vertreiben lassen; Thorspiegel sind selbst bei Rotglut beständig.

$\mathrm{Zu}$ den Reaktionen, welche den Thorspiegel als solchen identifizieren, seien nachstehende wiedergegeben:

Weder kaltes noch heißes Wasser greifen den Thorspiegel an.

Gelbes Ammoniumsulfid läßt ihn unverändert.

Kalte verdünnte Salzsäure bewirkt keine wesentlichen Veranderungen.

Heiße konzentrierte Salzsäure wirkt nur teilweise lösend.

Kalte verdünnte Salpetersäure reagiert wie Salzsäure.

Konzentrierte Salpetersäure wirkt nicht lösend, dagegen oxydierend.

Schwefelsäure reagiert wie Salzsäure.

Von Königswasser wird der Thorspiegel in der Wärme vollständig gelöst.

Schwefelwasserstoffgas schwärzt den Spiegel bei kräftiger Erhitzung infolge der Bildung des Sulfides. ${ }^{1}$ )

Salzsäuregas verwandelt das Sulfid in Metall, dabei ist gleichfalls starkes Erwärmen der Röhre notwendig. ${ }^{2}$ )

Alkalien sind ohne Einwirkung.

In nachstehender Tabelle sollen wie auf der Umseite die Reaktionen der spiegelbildenden Metalle verzeichnet werden:

1) Nilson, 1. c.

\%) Vgl. KRöss u. NiLson, l. c. 


\begin{tabular}{|c|c|c|c|c|c|c|c|}
\hline Metall & $\mathrm{HCl}$ & HNO, & $\begin{array}{c}\text { gelbes } \\
(\mathrm{NH}\end{array}$ & $\mathrm{NaOCl}$ & HCl-Gas & $\mathrm{H}_{3} \mathrm{~S}-\mathrm{Gas}_{\mathrm{B}}$ & $\mathrm{Cl}$ \\
\hline Arsen & gering & sofort & langeam & sofort & $\begin{array}{c}\text { nicht } \\
\text { merktich }\end{array}$ & gelb & weiB \\
\hline Antimon & " & $\because$ & gelöst & - & $n$ & Igelb bir rot & usrerändert \\
\hline Wismut & gelöst & $"$ & vertieft & - & $\begin{array}{c}\text { weib } \\
\text { langsam }\end{array}$ & braun & braun \\
\hline Zinn & $\begin{array}{l}\text { augen- } \\
\text { blicklich }\end{array}$ & $\begin{array}{c}\text { nicht } \\
\text { merklich }\end{array}$ & langsam & - & $\begin{array}{c}\text { 2agenblicklich } \\
\text { uwitat tbar }\end{array}$ & $\left|\begin{array}{c}\text { braun } \\
\text { sus } \mathrm{SnCl}_{3}\end{array}\right|$ & weiB \\
\hline Silicium & \multicolumn{3}{|c|}{ unlöslich } & \multicolumn{4}{|c|}{ unverăndert } \\
\hline Thorium & nicht $n$ & erklich & - & - & - & schwarz & - \\
\hline
\end{tabular}

Als Spezialreaktionen seien angeführt:

Die aus der Auflösung der Spiegel in Königswasser erhaltenen Chloride bzw. Nitrate benutzt man zum mikroskopischen Nachweis des Thoriums. Die Methoden sind von Haushofer ${ }^{1}$ ) ausgearbeitet worden. Aus der gereinigten Thorsalzlösung scheidet sich das Sulfat durch Hinzufügen von verdünnter Schwefelsäure in warzenförmigen Aggregaten $\mathrm{Th}\left(\mathrm{SO}_{4}\right)_{2}+8 \mathrm{H}_{2} \mathrm{O}$ ab. Durch Umkristallisieren gewinnt man leicht erkennbare, spitz pyramidale oder kurz prismatische Formen. Diese Reaktion wird vorteilhaft auf dem Objektträger vorgenommen.

Eine ebensogute dèr ersten an Eleganz nicht nachstehende Methode gelingt vermittelst Oxalsäure. Dabei benützt man zweckmäBig die neutrale oder schwach saure Thorsulfatlösung und behandelt tropfenweise mit Oxalsäure. Es entsteht bald ein kristallinischer Niederschalg, dessen Charakterisierung durch sechsseitige langgestreckte Täfelchen gegeben ist. Sternförmige und büschelförmige Bildungen sind gleichfalls nachweisbar.

Von den übrigen, für die Identifizierung des Thoriums noch in Frage stehenden Reaktionen ${ }^{2-9}$ ), kommen noch die Fällung des gelatinösen Thoriumhydroxydes mittels Alkalien oder Ammoniak, sowie die Bildung des Thoriumjodates mittels Jodsäure ${ }^{5}$ ) in Be-

1) K. HaUshofkR, Sitzungsber. d. bayr. Akad. 13, 436-448; J. B. 1884, 1551.

8) Bermurys, K. Vet. Acad. Handl. 1. c.

3) Graser, Journ. Amer. Chem. Soc. 18, 782; Chem.-Ztg. 20, 612-614; Chem. Centralbl. 1896, II, 803.

4) L. HABER, Monatsh. f. Chem. 18, 687; Chem. Centralbl. 1898,.I, 657.

5) R. J. MaYar, Z. anorg. Chem. 71 (1911), 65; Meyeh u. Spetwa, Chen.-Ztg. 85 (1910), 306. 
tracht. Die Jodsäurelösung kann durch Auflösen von $\mathrm{KJO}_{3}$ in Salpetersäure nach folgenden Angaben gewonnen werden:

I. $15 \mathrm{~g} \mathrm{KJO}_{3}+50 \mathrm{ccm}$ konz. $\mathrm{HNO}_{3} 1: 4$ and $100 \mathrm{~cm} \mathrm{H}_{2} \mathrm{O}$

II. $4 \mathrm{~g} \mathrm{KJO}+100 \mathrm{~cm}$ verd. $\mathrm{HNO}_{3} 3: 2$ und $400 \mathrm{ccm} \mathrm{H}_{2} \mathrm{O}$

Die Thorjodatreaktion kann in der Zersetzungsröhre selbst vorgenommen werden und liefert bei vorsichtiger Arbeit stets positive Resultate. Man macht für diese Zwecke die Jodsäurelösung stark salpetersauer (die Lösung als auch die Röhre müssen frei von Salzsäure sein) und kocht im Proberohr den durch Salpetersäure oxydierten, in Schwefelsäure aufgelösten Spiegel einige Minuten. Der Thorspiegel erscheint dann in gelber Farbe, ist im Überschuß des Fällungsmittels sowie in Oxalsäure löslich, was ihn sofort von Zirkon unterscheidet, dessen Jodat in Oxalsäure unlöslich ist.

Ebenso wie Zinn gibt auch Thorium zum Unterschied von Wismut und Antimon keine Lumineszenzreaktion, wenn man in der Wasserstoffflamme geglühtes reinstes Calciumoxyd in die Thorwasserstoffflamme hält. ${ }^{1-2}$ ) In Königswasser gelöste Spiegelsubstanz wirkt gleichfalls negativ.

Füx den raschen Nachweis des Thoriums in der Zersetzungsröhre können demnach die Unlöslichkeit in verdünnten Säuren bzw. Löslichleit in Königswasser neben den letztangeführten mikroskopischen Reaktionen herangezogen werden.

Ein wesentlich günstigeres Verfahren zur Gewinnung von Thorspiegeln beruht auf der Kondensation und Wiederverflüchtigung des Thoriumwasserstoffes mittels flüssiger Luft. Wir bedienten uns vorteilhaft eines Schlangenkühlers nach CL. WinkLER, welcher, in ein becherförmiges WeinhoLDsches Gefäß mit flüssiger Luft eingetaucht, die Kondensation des Thorwasserstoffes bewirkte. Das Kondensationsgefäß wurde an das Pentoxydrohr und die Zersetzungsröhre geschaltet und mit zwei Glashähnen versehen, worauf mit dem Eintragen der Reaktionsmasse begonnen wurde. Hinter der Frhitzungsstelle setzte sich nunmehr kein Spiegel ab, was darauf zurückzuführen war, daß die Dampfspannung des verflüssigten Thorwasserstoffes nicht mehr langte, um das Entweichen des Gases

1) J. Donau, Monatsh. 34 (1913), 949. - Die Arbeitsmethoden der Mikrochemie, Stuttgart 1913, 18 u. 30.

2) Paneth u. Winternitz, 1. c., 1736. 
zu ermöglichen. Bei ungenügender Kühlung, d. h. wenn das WEINHouDsche Gefäß nicht vollkommen mit flüssiger Luft gefüllt war, erschien sofort ein brauner Ring von Thorium unweit der Erhitzungsstelle. Nachdem der Schlangenkühler aus der flüssigen Luft gehoben wurde, entstand sofort ein kräftiger Thorspiegel beiderseits, ja sogar innerhalb der Erhitzungsstelle von wechselnder Farbe von Braun bis Grau. Der verflüssigte Teil des Thorwasserstoffes konnte nur kurze Zeit verschlossen im Kühlgefä $B$ aufbewahrt werden, da er sehr unbeständig ist. Die Nachprüfung der hypothetischen Formel $\mathrm{ThH}_{4}$ dürfte somit mit einigen Schwierigkeiten verbunden sein.

Der Einwand, wonach es sich bei den ron uns erhaltenen Spiegeln um Abscheidungen von Thor C oder Thor B handelt, wird sofort entkräftet, wenn man bedenkt, da $B$ diese in minimaler Menge gebildet werden, aber auch ihre Reaktionen mit Wismut bzw. Blei identisch sein müßten, was jedoch keineswegs, wie aus den vorhergehenden Erörterungen klar hervorgeht, der Fall ist. ${ }^{1}$ )

Orientierende Vorversuche über die Radioaktivität des Thorwasserstoffes ergaben nur geringe, wahrscheinlich von $\alpha$-Strahlen herrührende Ionisation im Emanationsraum des Elektrometers.

Zum Schluß sei noch auf die Tatsache hingewiesen, wonach der Thoriumwasserstoff das schwerste unter normalen Bedingungen gebildete Gas ist, was auch mit den von $\mathrm{P}_{\text {ANETH }}{ }^{2}$ ) bei Poloniumund Wismutwasserstoff gefundenen Daten in Einklang steht. Der durch Rechnung ermittelte Wert für die Dichte des gasförmigen Thorhydrides beträgt $236,15 \quad\left(0_{2}=32\right)$, welche Zahl wohl auch die Dichte der Radiumemanation 222 übertreffen wird.

Anhangsweise sei noch einiges über die Theorie der Spiegelbildung, sowie die daraus zu ziehenden Folgerungen dargetan. KoHLSCHÜтTER ${ }^{3}$ ) vertritt die Anschauung, wonach sich das Element im Momente der Abseheidung im atomaren Zustand im Wasserstoff schwebend befindet, und verwendet hierfür den Ausdruck „Pseudogaszustand". Eis liegt hier der seltene Fall vor, wo sich das Atom im Momente der Zersetzung der Wasserstoffverbindung im ,Status nascens" befindet, somit energetischen Einflüssen geringeren Widerstand entgegensetzen wird als dies in molekularer Form zutreffend

1) F. Paneth, Ber. d. Wien. Akad. 127 (1918), $1732-1745$.

2) Ebenda, 1756.

3) KoHLSCHÜTTER, Z. Elektrochem. 18 (1912), 837-_838. 
816 A. Klauber 2. J. Mell v. Hellonheim. Gasförmiges Hydrid von Thorium

ist. Durch passende Wahl der Versuchsanordnung ist somit die Möglichkeit geschaffen, das Element im Momente des Entstehens anzugreifen und die Zersplitterung des Atomes zu bewirken. Dadurch eröffnet sich für die von RUTHERFORD ${ }^{1}$ ) angebahnte Forschung ein aussichtsreiches Feld und wir behalten uns weitere diesbetreffende Mitteilungen vor.

1) RUTheRYokd, Phil. Mag. 87 (1919), 729.

Prag-Smichow, Wissenschaftliches Labaratorium, im Juli 1920.

Bei der Redaktion eingegangen am 7. August 1920.

\section{Druckfehlerberichtignng:}

Band 112, Heft 2 und 3, S. 221, Darstellung des periodiochen Systems der Elemente von G. Schaltmmasid, Anmorkung 1) muB heiBen: Chascourtom. Die tellurische Helix, und weiter Crookns, Lominiskaten-Kurve. 